\title{
Scattering by Dust in Planetary Nebulae: Polarization as a Diagnostic Tool
}

\author{
J. R. Walsh \\ Space Telescope European Co-ordinating Facility, European Southern \\ Observatory, Karl-Schwarzschild Strasse 2, D-85748 Garching, \\ Germany \\ L. Walsh \\ Radiobiological Institute, Ludwig-Maximilian University, Schiller Strasse \\ 42, D-80336 Munich, Germany
}

There is a wealth of evidence for the presence of dust in PN from continuum and line emission in the IR, spatially varying extinction and ERE emission in the optical and strength of resonance lines in the UV. The dust emits strongly in the IR but in the UV-optical absorbs and scatters the radiation from the central star and the gaseous emission envelope. Linear polarization of PN light is thus an expected consequence of the presence of dust both within and outside the ionized region. Intrinsic scattering haloes of $\mathrm{PN}$ can be confused with local (telescope + atmospheric) scattering; polarimetry however allows the morphology of a dusty halo to be studied. Spectropolarimetry of emission line profiles allows the kinematics of the dust relative to the gaseous emission to be uniquely studied. Polarization contributes to understanding the scattering properties of the grains in PN and how they relate to those of the general ISM to which they will subsequently contribute.

Linear spectropolarimetry of strong emission lines in six planetary nebulae has been observed to study the velocity structure of the linear polarization profiles. Observations were obtained with the William Herschel Telescope, ISIS spectrometer and polarization module with half-wave plate at selected positions both over the central bright nebulae and offset positions in the lower surface brightness outer regions. The detection of a dust scattering halo around NGC 7027 was reported by Walsh \& Clegg (1994) and polarized wings to [O III] and $\mathrm{H} \alpha$ line profiles were detected over the bright core. From observations of five other sources, only one target, NGC 2392, showed no evidence for polarized line profile wings.

The behaviour of the line profiles can be understood in terms of a radially expanding emission and dust nebula. In this configuration a dust grain reflects line emission towards the observer and, on account of the recession velocity of the emission line relative to the grain, the scattered emission line profile is shifted to the red wing of the line, as observed. Thus the presence of a red wing to an emission line (either forbidden or recombination) may not necessarily signify high (gas) velocities; spectropolarimetry is required to confirm its origin. Detailed modelling of the kinematics of the gas and dust can reveal the expansion pattern for the dust, which may not be coupled to the ionized gas. 

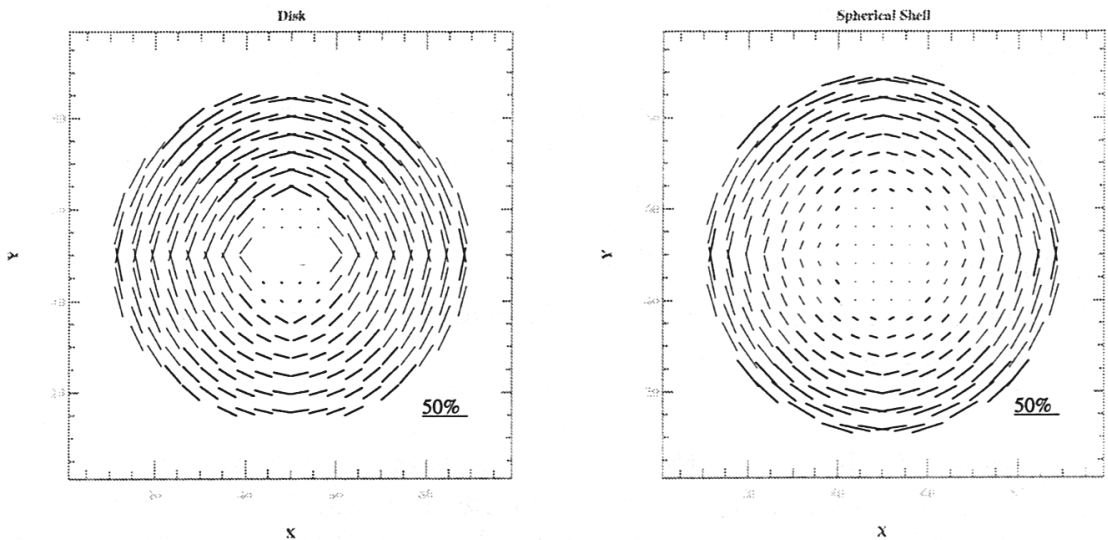

Figure 1. Model polarization vector maps of the single scattering dust nebulae around a prolate ellipsoidal shell emission nebula with major axis tilted by $30^{\circ}$ to the line of sight. The left map is for an equatorial disc concentric with the nebular long axis; the right map for a spherical shell. Note the differences between the maps such as the larger polarization across the centre for the disc and the increase of polarization outwards for the spherical shell dust nebula. The dust is amorphous carbon (Rouleau \& Martin, 1991, $\mathrm{BE} 1)$ with a size distribution $\mathrm{N} \propto a^{-3.5}$, with $\mathrm{a}_{\min }=0.005, \mathrm{a}_{\max }=0.025 \mu \mathrm{m}$ (Mathis et al., 1977 (MRN) law).

The potential of imaging polarimetry for the study of dust in PN has been demonstrated through 3D geometrical and dust scattering models. The models use Mie theory to predict the scattering properties of the grain size distribution and 3D geometrical forms for the emitting gas and the dust. Single and multiple scattering can be considered. Comparison of model polarization maps with observed ones can allow the 3D form of the dust halo to be inferred. The figure shows how two forms of dust nebula, surrounding an ellipsoidal shell emission nebula (based on the model of $\mathrm{BD}+30^{\circ} 3639$ from HST observations, Harrington et al., 1997), can be distinguished from polarization vector maps.

\section{References}

Harrington, J. P., Lame, N. J., White, S. M., Borkowski, K. J., 1997, AJ, 113, 2147

Mathis, J. S., Rumpl, W., Nordsieck, K. H., 1977, ApJ, 217, 425

Rouleau, F., \& Martin, P. G., 1991, ApJ, 377, 526

Walsh, J. R., \& Clegg, R. E. S., 1994, MNRAS, 268, L41 